\title{
A MOD TWO ANALOGUE OF A CONJECTURE OF COOKE
}

\author{
J. Aguadé, C. Broto and D. Notbohm
}

$\S 0$. Introduction.

The mod two cohomology of the three connective covering of $S^{3}$ has the form

$$
\mathbb{F}_{2}\left[x_{2 n}\right] \otimes E\left(S q^{1} x_{2 n}\right)
$$

where $x_{2 n}$ is in degree $2 n$ and $n=2$. If $F$ denotes the homotopy theoretic fibre of the map $S^{3} \rightarrow B^{2} S^{1}$ of degree 2 , then the mod 2 cohomology of $F$ is also of the same form for $n=1$. Notice (cf. section 7 of the present paper) that the existence of spaces whose cohomology has this form for high values of $n$ would immediately provide Arf invariant elements in the stable stem. Hence, it is worthwhile to determine for what values of $n$ the above algebra can be realized as the mod 2 cohomology of some space. The purpose of this paper is to construct a further example of a space with such a cohomology algebra for $n=4$ and to show that no other values of $n$ are admissible. More precisely, we prove:

Theorem 1. There is a space $X$ such that $H^{*}\left(X ; \mathbb{F}_{2}\right) \cong \mathbb{F}_{2}\left[x_{2 n}\right] \otimes E\left(S q^{1} x_{2 n}\right)$ with $x_{2 n}$ of degree $2 n$ if and only if $n=1,2,4$.

The "only if" part of theorem 1 can be considered, after $[\mathbf{3}]$ and $[\mathbf{4}]$, the mod two version of what we call there the Cooke conjecture. Its proof is similar to the proof for odd primes (which is contained in [3]), but one should be slightly more careful in small degrees. The most interesting goal of this paper is probably the construction of what we think to be a remarkable space $X$ with $H^{*}\left(X ; \mathbb{F}_{2}\right) \cong \mathbb{F}_{2}\left[x_{8}\right] \otimes E\left(S q^{1} x_{8}\right)$ and $\operatorname{deg} x_{8}=8$.

This paper represents a part of the $p=2$ case of the general problem of the homotopy classification of spaces realizing cohomology algebras of the type $\mathbb{F}_{p}[x] \otimes E(\beta x)$ where $\beta$ denotes the Bockstein homomorphism. The case of $p$ odd was completely elucidated in [3]. The homotopy classification in the case $p=2$ should appear in [4]. In particular, as any reader familiar with $[\mathbf{3}]$ would expect, there are infinitely many different 2 -complete spaces realizing each cohomology algebra in the theorem and the proof of the homotopy classification theorem will not be short. The present paper has been written in a self contained way, and can be read independently of [3] and [4], although it may be helpful to be familiar with the techniques discussed there.

Since the technical details of the construction of a space $X$ with $H^{*}\left(X ; \mathbb{F}_{2}\right) \cong \mathbb{F}_{2}\left[x_{8}\right] \otimes$ $E\left(S q^{1} x_{8}\right)$ may look involved, we provide now an outline of the way in which $X$ can be reached. Let $F$ be the homotopy theoretic fibre of the degree two map from $S^{3}$ to $B^{2} S^{1}$ (as

J. Aguadé and C. Broto are partially supported by DGICYT grant PB91-0467

Typeset by $\mathcal{A M S}_{\mathcal{S}}-\mathrm{TEX}_{\mathrm{E}}$ 
noted above, $F$ turns out to provide a space as in the theorem for $n=1)$ and let $W$ be the homotopy quotient of $F$ by an involution coming from a degree -1 map of $S^{3}$ (see Section 1 ). Then one has a map $f: B D \rightarrow W$ where $D$ is the dihedral group of order 8 . Using the techniques developed by Lannes $([\mathbf{8}])$ and other authors one proves that the function space $\operatorname{map}(B D, W)_{f}$ is $\bmod 2$ homotopy equivalent to $B \mathbb{Z} / 2 \times S^{2}$. From this fact, one can construct a diagram which, up to homotopy, looks like

$$
G L_{2}\left(\mathbb{F}_{2}\right) \quad \circlearrowright \quad B V \times S^{2} \quad \Longrightarrow W
$$

where $V$ denotes the group of diagonal matrices in $D$. Let $Y$ be the homotopy colimit of this diagram. Then the space $X$ we are looking for can be taken as the 2-connective covering of $Y$.

The steps towards the construction of $X$ appear in sections 1 to 5 . Section 6 contains the proof of the "only if" part of the theorem, i.e. the Cooke conjecture. A final section contains some inconclusive remarks related to the Arf invariant.

We work exclusively at the prime 2 and mod 2 coefficients are implicitly assumed. When describing cohomology generators the subscripts usually denote the degrees of the generators.

The first and second author would like to thank the Sonderforschungsbereich 170 in Göttingen for the support during part of this joint work.

\section{$\S 1$. The SPACE $W$.}

We start with the action of $\mathbb{Z} / 2$ on $S^{1}$ given by the extension $S^{1} \rightarrow O(2) \rightarrow \mathbb{Z} / 2$. A functorial classifying space construction (for groups see [10] and for $A_{\infty}$-spaces [12] leads to a cellular action of $\mathbb{Z} / 2$ on $B S^{1}$ and then on $E B S^{1}$ and $B^{2} S^{1}$. Moreover the projection $E B S^{1} \rightarrow B^{2} S^{1}$ is equivariant and the action on $B S^{1}$ is reproduced on the fibre over the base point of $B^{2} S^{1}$. The sphere $S^{3}$ is the first homotopy theoretic fibrecell of $B^{2} S^{1}$ and inherits an action of $\mathbb{Z} / 2$ which turns out to be the two fold suspension of the original action on $S^{1}$. The composition of an equivariant degree two map $S^{3}=\Sigma^{2} S^{1} \rightarrow \Sigma^{2} S^{1}=S^{3}$ with the inclusion of the 3 -cell gives a concrete equivariant degree two map $S^{3} \rightarrow B^{2} S^{1}$. Now $F$ is defined by the pull back diagram

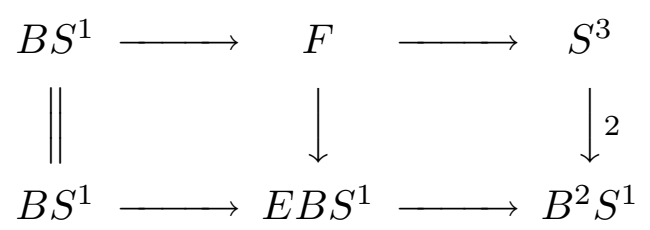

so that it is given a $\mathbb{Z} / 2$ action that makes equivariant all maps in the diagram. Recall that $H^{*}(F)=\mathbb{F}_{2}\left[x_{2}\right] \otimes E\left(S q^{1} x_{2}\right)$, where $x_{2}$ is detected in $B S^{1}$ and $S q^{1} x_{2}$ is the spherical class that comes from $S^{3}$.

Definition 2. Let $W$ denote the homotopy quotient of $F$ by the action of $\mathbb{Z} / 2$ :

$$
W=F_{h \mathbb{Z} / 2}=E \mathbb{Z} / 2 \times_{\mathbb{Z} / 2} F
$$


The rest of the section is devoted to the computation of the cohomology of $W$. Observe that this space fits in the diagram

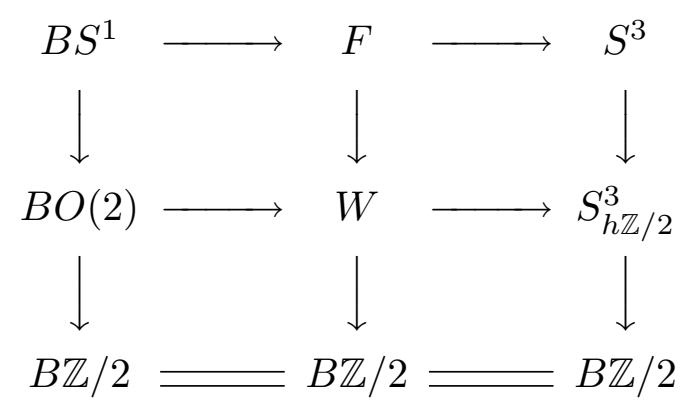

where the columns are fibrations and all of them have a section provided by the base point of $B S^{1}, F$ or $S^{3}$ which is fixed under the action of $\mathbb{Z} / 2$.

Proposition 3. $H^{*}\left(S_{h \mathbb{Z} / 2}^{3}\right) \cong \mathbb{F}_{2}\left[w_{1}\right] \otimes E\left(y_{3}\right)$, with $S q^{1}\left(y_{3}\right)=w_{1} y_{3}$ and $S q^{i}\left(y_{3}\right)=0$ for $i \geq 2$.

Proof. Our action of $\mathbb{Z} / 2$ on $S^{1}$ is already the suspension of the action of $\mathbb{Z} / 2$ on itself. It follows that the fibration $S^{3} \rightarrow S_{h \mathbb{Z} / 2}^{3} \rightarrow B \mathbb{Z} / 2$ is the sphere bundle of a four dimensional real bundle $\xi$ over $B \mathbb{Z} / 2$, obtained as the Whitney sum of the universal line bundle

$$
\mathbb{R} \rightarrow E \mathbb{Z} / 2 \times_{\mathbb{Z} / 2} \mathbb{R} \rightarrow B \mathbb{Z} / 2
$$

and a three dimensional trivial bundle. Hence the Stiefel-Whitney classes of $\xi$ are $w_{1}(\xi)=$ $w_{1} \in \mathbb{F}_{2}\left[w_{1}\right] \cong H^{*}(B \mathbb{Z} / 2)$ and $w_{i}(\xi)=0$ if $i>1$.

Let $T(\xi) \simeq \operatorname{Cofibre}\left(S_{h \mathbb{Z} / 2}^{3} \rightarrow B \mathbb{Z} / 2\right)$ be the Thom space. Since the action of the Steenrod squares on the Thom class is given by multiplication by the Stiefel-Whitney classes, the complete structure of $H^{*}\left(S_{h \mathbb{Z} / 2}^{3}\right)$ follows from the split exact sequence of $H^{*}(B \mathbb{Z} / 2)$ $\mathcal{U}$-modules

$$
0 \rightarrow H^{*}(B \mathbb{Z} / 2) \rightarrow H^{*}\left(S_{h \mathbb{Z} / 2}^{3}\right) \stackrel{\Delta}{\longrightarrow} \widetilde{H}^{*+1}(T(\xi)) \rightarrow 0
$$

Proposition 4. $H^{*}(W) \cong \mathbb{F}_{2}\left[w_{1}, w_{2}\right] \otimes E\left(y_{3}\right)$ with $S q^{1} w_{2}=w_{1} w_{2}+y_{3}, S q^{1} y_{3}=w_{1} y_{3}$ and $S q^{i} y_{3}=0$ if $i \geq 2$. The maps induced in cohomology by the diagram (2) are as suggested by the names of the generators.

Proof. We compute $H^{*}(W)$ by means of the Serre spectral sequence for the fibration $F \rightarrow W \rightarrow B \mathbb{Z} / 2$. Since $\mathbb{Z} / 2$ can only act trivially on $H^{*}(F)$ we have

$$
E_{2}^{*, *} \cong H^{*}(B \mathbb{Z} / 2) \otimes H^{*}(F) \cong \mathbb{F}_{2}[u] \otimes \mathbb{F}_{2}\left[x_{2}\right] \otimes E\left(S q^{1} x_{2}\right) \Rightarrow H^{*}(W)
$$

It is clear that $x_{2}$ is transgressive and then so is $S q^{1} x_{2}$. But the fibration has a section and therefore $\mathbb{F}_{2}[u]$ survives to $E_{\infty}^{*, *}$. Hence the possible differentials on $x_{2}$ and $S q^{1} x_{2}$ are zero and the spectral sequence collapses at the $E_{2}$-term. That is:

$$
G r H^{*}(W) \cong E_{\infty}^{*, *} \cong E_{2}^{*, *} \cong \mathbb{F}_{2}\left[u, x_{2}\right] \otimes E\left(S q^{1} x_{2}\right)
$$


If we look at the Serre spectral sequences for the left and right columns fibrations of diagram (2) the above argument shows:

$$
\begin{aligned}
& G r H^{*}(B O(2)) \cong \mathbb{F}_{2}\left[u, x_{2}\right] \\
& G r H^{*}\left(S_{h \mathbb{Z} / 2}^{3}\right) \cong \mathbb{F}_{2}[u] \otimes E\left(S q^{1} x_{2}\right)
\end{aligned}
$$

and the maps $G r H^{*}(B O(2)) \leftarrow G r H^{*}(W) \leftarrow G r H^{*}\left(S_{h \mathbb{Z} / 2}^{3}\right)$ relate the generators with the same names. It is well known the structure of $H^{*}(B O(2))$ and we know that of $H^{*}\left(S_{h \mathbb{Z} / 2}^{3}\right)$ from Proposition 3. It follows the existence of polynomial generators $w_{1}$ and $w_{2}$ in $H^{*}(W)$ that map to the generators of $H^{*}(B O(2))$ and an exterior generator $y_{3}$ that comes from the one in $H^{*}\left(S_{h \mathbb{Z} / 2}^{3}\right)$. Hence $H^{*}(W)=\mathbb{F}_{2}\left[w_{1}, w_{2}\right] \otimes E\left(y_{3}\right)$. Finally, the action of the Steenrod algebra on $H^{*}(W)$ is forced as well by the maps $B O(2) \rightarrow W \rightarrow S_{h \mathbb{Z} / 2}^{3}$ and $F \rightarrow W$.

\section{§2. Preliminary Results}

Before starting the computations in which we are interested we recall in this section some results about general mapping spaces which will be needed in the next section.

2.1. Let $P$ be a finite $p$ group and $G$ a compact Lie group. The classifying space construction induces a bijection $([\mathbf{7}])$

$$
\operatorname{Rep}(P, G) \cong[B P, B G]
$$

Furthermore, for a given representation $\rho: P \rightarrow G$, if $C_{\rho}(P)$ denotes the centralizer of the image of $\rho$, we have a homomorphism $P \times C_{\rho}(P) \rightarrow G$ and $B P \times B C_{\rho}(P) \rightarrow B G$ whose adjoint

$$
B C_{\rho}(P) \rightarrow \operatorname{map}\left(B P, B G_{2}^{\widehat{n}}\right)_{B \rho}
$$

is a $\bmod -p$ equivalence, i.e. induces an isomorphism in mod-p cohomology.

2.2. For an elementary abelian $p$ group $V$ and a $p$-complete space of finite type, there is a bijection $([8])$

$$
[B V, X] \cong \operatorname{Hom}_{\mathcal{K}}\left(H^{*}(X), H^{*}(B V)\right)
$$

where $\mathcal{K}$ denotes the category of unstable algebras over the Steenrod algebra. Furthermore, the functor $T_{V}: \mathcal{K} \rightarrow \mathcal{K}$, left adjoint to tensoring with $H^{*}(B V)$ computes cohomology of mapping spaces. If $R$ is an object of $\mathcal{K}$ we write $T_{V}(R) \cong \coprod_{f \in \operatorname{Hom}_{\mathcal{K}}\left(H^{*}(B V), R\right)} T_{V}(R, f)$ as a sum of connected components. Then for a given map $f: B V \rightarrow X$, there is an isomorphism of algebras over the Steenrod algebra

$$
T_{V}\left(H^{*}(X), f^{*}\right) \cong H^{*}\left(\operatorname{map}(B V, X)_{f}\right)
$$

provided $T_{V}\left(H^{*}(X), f^{*}\right)$ is of finite type and free in d egrees $\leq 2$. Recall that for $p=2$ Lannes called a $\mathrm{n}$ object $R$ of $\mathcal{K}$ free in degrees $\leq 2$ if the kernel of the multiplication $R^{1} \otimes R^{1} \rightarrow R^{2}$ is generated by elements of the form $x \otimes y+y \otimes x$.

Moreover there is a coaugmentation $\varepsilon \rightarrow T_{V}$ that under the above isomorphism corresponds to the map in cohomology induced by the evaluation $\operatorname{map}(B V, X)_{f} \rightarrow X$.

Finally, we collect some concrete computations that we use later. The proof is along the lines of $[\mathbf{4}]$ section 3 . 
Lemma 5. (1) Let $s^{*}: H^{*}\left(S_{h \mathbb{Z} / 2}^{3}\right) \cong \mathbb{F}_{2}\left[w_{1}\right] \otimes E\left(y_{3}\right) \rightarrow H^{*}(B \mathbb{Z} / 2)$ be the projection onto the polynomial part and $g^{*}: H^{*}\left(S_{h \mathbb{Z} / 2}^{3}\right) \rightarrow H^{*}(B V), V=\mathbb{Z} / 2 \times \mathbb{Z} / 2$, the composition of $s^{*}$ and the diagonal map, then

$$
T_{\mathbb{Z} / 2}\left(H^{*}\left(S_{h \mathbb{Z} / 2}^{3}\right), s^{*}\right) \cong T_{V}\left(H^{*}\left(S_{h \mathbb{Z} / 2}^{3}\right), g^{*}\right) \cong \mathbb{F}_{2}\left[w_{1}\right] \otimes E\left(y_{2}\right)
$$

with $S q^{i} y_{2}=0$ for $i \geq 1$. The coaugmentation is the inclusion given by $w_{1} \mapsto w_{1}$ and $y_{3} \mapsto w_{1} y_{2}$

(2) Let $f^{*}: H^{*}(W) \rightarrow H^{*}(B V), V=\mathbb{Z} / 2 \times \mathbb{Z} / 2$, be the composition $\mathbb{F}_{2}\left[w_{1}, w_{2}\right] \otimes E\left(y_{3}\right) \rightarrow$ $\mathbb{F}_{2}\left[w_{1}, w_{2}\right] \rightarrow \mathbb{F}_{2}\left[t_{1}, t_{2}\right]$, then

$$
T_{V}\left(H^{*}(W), f^{*}\right) \cong \mathbb{F}_{2}\left[t_{1}, t_{2}\right] \otimes E\left(y_{2}\right)
$$

Moreover, the map $T_{V}\left(H^{*}(W), f^{*}\right) \rightarrow T_{V}\left(H^{*}(B O(2)), B i^{*}\right)$ induced by $B O(2) \rightarrow W$ is the projection $\mathbb{F}_{2}\left[t_{1}, t_{2}\right] \otimes E\left(y_{2}\right) \rightarrow \mathbb{F}_{2}\left[t_{1}, t_{2}\right]$.

\section{$\S 3$. Some Mapping SPACES}

¿From now on all spaces are assumed to be completed at the prime two.

Let $D$ denote the dihedral group of order eight. We represent it as the subgroup $i: D \hookrightarrow$ $O(2)$ of $O(2)$ generated by the matrices

$$
\left(\begin{array}{cc} 
\pm 1 & 0 \\
0 & \pm 1
\end{array}\right) \quad \text { and } \quad\left(\begin{array}{ll}
0 & 1 \\
1 & 0
\end{array}\right)
$$

Let $V$ denote the subgroup given by the diagonal matrices, and let $Z:=Z(O(2)) \cong$ $Z(D) \cong \mathbb{Z} / 2$ be the center of $O(2)$ or of $D$.

We are interested in the mapping space $\operatorname{map}(B D, W)_{f}$ where $f$ is the composition $B D \rightarrow B O(2) \rightarrow W$.

Interpreting the top row fibration in diagram (1), $B S^{1} \rightarrow F \rightarrow S^{3}$, as a pull back diagram and applying the Borel construction $-\times_{\mathbb{Z} / 2} E \mathbb{Z} / 2$ yields another pull back diagram

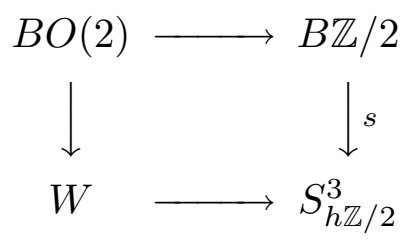

And then we apply the functor $\operatorname{map}(B D,-)$ to get the diagram

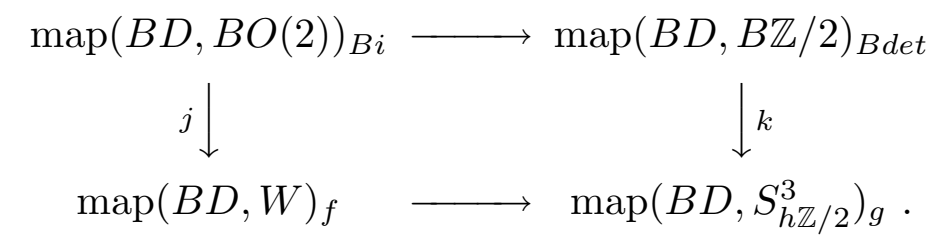

To get a pull back diagram, one might have to take more components than just the one given by $B i$ in the upper left corner. Let $B j: B D \rightarrow B O(2)$ be a lift of $f$ (see 2.1). The 
restriction $\left.B j\right|_{B V}$ is homotopic to $\left.B i\right|_{B V}$. This follows by 2.2 from looking at the induced maps in cohomology. There also exists only one extension of $i: V \rightarrow O(2)$ to $D$. Hence, $B j$ and $B i$ are homotopic and the above diagram is a pull back diagram.

The homology of the mapping spaces of the top row is calculated. In the composition

$$
B Z \rightarrow \operatorname{map}(B D, B O(2))_{B i} \rightarrow \operatorname{map}(B D, B \mathbb{Z} / 2)_{B d e t} \simeq B \mathbb{Z} / 2
$$

the first arrow is a homotopy equivalence by 2.1. The second map is induced by the determinant and therefore trivial. The homotopy theoretic fibre is homotopy equivalent to $\mathbb{Z} / 2 \times B \mathbb{Z} / 2$.

Next, we calculate $\operatorname{map}\left(B D, S_{h \mathbb{Z} / 2}^{3}\right)_{g} \cdot S^{2}$ is the fix point set of $S^{3}$ under the action of $\mathbb{Z} / 2$, hence the section $s$ extends to an inclusion $l: B \mathbb{Z} / 2 \times S^{2} \rightarrow S_{h \mathbb{Z} / 2}^{3}$. Now we can use the factorization of $g: B D \rightarrow S_{h \mathbb{Z} / 2}^{3}$ as $B D \stackrel{B \text { det }}{\longrightarrow} B \mathbb{Z} / 2 \stackrel{s}{\rightarrow} S_{h \mathbb{Z} / 2}^{3}$ in order to define a map

$$
B D \times B \mathbb{Z} / 2 \times S^{2} \stackrel{B \text { det }}{\longrightarrow} B \mathbb{Z} / 2 \times B \mathbb{Z} / 2 \times S^{2} \stackrel{m}{\longrightarrow} B \mathbb{Z} / 2 \times S^{2} \stackrel{l}{\rightarrow} S_{h \mathbb{Z} / 2}^{3}
$$

which coincides with $g$ when restricted to $B D$ and with $l$ when restricted to $B \mathbb{Z} / 2 \times S^{2}$. Let

$$
h: B \mathbb{Z} / 2 \times S^{2} \rightarrow \operatorname{map}\left(B D, S_{h \mathbb{Z} / 2}^{3}\right)_{g}
$$

be the adjoint of that composition. Then we have:

Proposition 6. (1) $h: B \mathbb{Z} / 2 \times S^{2} \rightarrow \operatorname{map}\left(B D, S_{h \mathbb{Z} / 2}^{3}\right)_{g}$ is a homotopy equivalence.

(2) The evaluation $\operatorname{map}\left(B D, S_{h \mathbb{Z} / 2}^{3}\right)_{g} \rightarrow S_{h \mathbb{Z} / 2}^{3}$ induces in cohomology the map given by $\omega_{1} \mapsto \omega_{1}$ and $y_{3} \mapsto \omega_{1} y_{2}$. Here, we write $H^{*}(B \mathbb{Z} / 2) \cong \mathbb{F}_{2}\left[w_{1}\right]$ and $H^{*}\left(S^{2}\right) \cong E\left(y_{2}\right)$.

Proof. Since the dihedral group sits in a split extension

$$
V \rightarrow D \rightarrow \Sigma_{2}
$$

the computation of $\operatorname{map}\left(B D, S_{h \mathbb{Z} / 2}^{3}\right)$ reduces to the computation of the homotopy fixed point set by the action of $\Sigma_{2}$ of $\operatorname{map}\left(\widetilde{B V}, S_{h \mathbb{Z} / 2}^{3}\right)$, where $\widetilde{B V}=E D / V$ (see [11], remark $3.12)$ :

$$
\operatorname{map}\left(B D, S_{h \mathbb{Z} / 2}^{3}\right) \simeq \operatorname{map}\left(\widetilde{B V}, S_{h \mathbb{Z} / 2}^{3}\right)^{h \Sigma_{2}}
$$

Recall that for a $G$-space $X$ the homotopy fixed point set $X^{h G}$ is defined as the space of equivariant maps $\operatorname{map}_{G}(E G, X)$ and coincides with the space of lifts of the fibration $X \times_{G} E G \rightarrow B G$.

So $\operatorname{map}\left(B D, S_{h \mathbb{Z} / 2}^{3}\right)_{f}$ is homotopic to a component of $\operatorname{map}\left(\widetilde{B V}, S_{h \mathbb{Z} / 2}^{3}\right)^{h \Sigma_{2}}$. Observe that $g: \widetilde{B V} \rightarrow S_{h \mathbb{Z} / 2}^{3}$, defined as the composition $\widetilde{B V} \rightarrow B D \stackrel{g}{\rightarrow} S_{h \mathbb{Z} / 2}^{3}$, also factors as

$$
\widetilde{B V} \stackrel{B \text { det }}{\longrightarrow} B \mathbb{Z} / 2 \stackrel{s}{\rightarrow} S_{h \mathbb{Z} / 2}^{3}
$$

and $B$ det is $\Sigma_{2}$-equivariant with trivial action of $\Sigma_{2}$ on $B \mathbb{Z} / 2$. We have therefore a $\Sigma_{2^{-}}$ equivariant map

$$
\operatorname{map}\left(B \mathbb{Z} / 2, S_{h \mathbb{Z} / 2}^{3}\right)_{s} \rightarrow \operatorname{map}\left(\widetilde{B V}, S_{h \mathbb{Z} / 2}^{3}\right)_{g}
$$


with trivial action of $\Sigma_{2}$ on the left hand side. Furthermore, by 2.2 this map is a homotopy equivalence and so it induces a homotopy equivalence

$$
\operatorname{map}\left(B \mathbb{Z} / 2, S_{h \mathbb{Z} / 2}^{3}\right)_{s} \rightarrow \operatorname{map}\left(\widetilde{B V}, S_{h \mathbb{Z} / 2}^{3}\right)_{g}^{h \Sigma_{2}}
$$

On the other hand, $l: B \mathbb{Z} / 2 \times S^{2} \rightarrow S_{h \mathbb{Z} / 2}^{3}$ induces

$$
B \mathbb{Z} / 2 \times S^{2} \rightarrow \operatorname{map}\left(B \mathbb{Z} / 2, B \mathbb{Z} / 2 \times S^{2}\right)_{i_{1}} \rightarrow \operatorname{map}\left(B \mathbb{Z} / 2, S_{h \mathbb{Z} / 2}^{3}\right)_{s}
$$

where $i_{1}: B \mathbb{Z} / 2 \rightarrow B \mathbb{Z} / 2 \times S^{2}$ is the inclusion of the first factor so that $s=k \circ i_{1}$. Observe that the first arrow is a homotopy equivalence and also the second one by 2.2. Finally, one checks that the composition

$$
B \mathbb{Z} / 2 \rightarrow \operatorname{map}\left(B \mathbb{Z} / 2, S_{h \mathbb{Z} / 2}^{3}\right)_{s} \rightarrow \operatorname{map}\left(\widetilde{B V}, S_{h \mathbb{Z} / 2}^{3}\right)_{g}^{\Sigma_{2}} \simeq \operatorname{map}\left(B D, S_{h \mathbb{Z} / 2}^{3}\right)_{g}
$$

is the map $h$. This proves (1). Part (2) of the proposition follows by 2.2 .

This statement calculates the mapping space in the lower right corner of diagram (4). Collecting all the facts we get a mod-2 pull back diagram

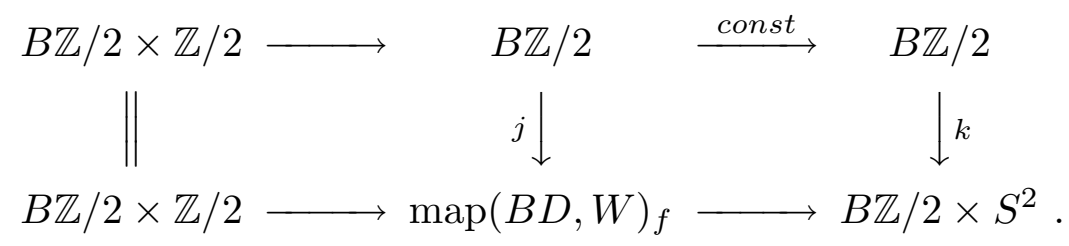

from which we can calculate $\operatorname{map}(B D, W)_{f}$. Observe that $k: B \mathbb{Z} / 2 \rightarrow B \mathbb{Z} / 2 \times S^{2}$ is the inclusion of the first factor. This follows by construction of the equivalences $B \mathbb{Z} / 2 \stackrel{\simeq}{\longrightarrow}$ $\operatorname{map}(B D, B \mathbb{Z} / 2)_{B \text { det }}($ cf. 2.1$)$ and $h: B \mathbb{Z} / 2 \times S^{2} \stackrel{\simeq}{\longrightarrow} \operatorname{map}\left(B D, S_{h \mathbb{Z} / 2}^{3}\right)_{g}$.

Proposition 7. The mapping space $\operatorname{map}(B D, W)_{f}$ is homotopy equivalent to $B \mathbb{Z} / 2 \times S^{2}$.

Proof. First we claim that $j$ in diagram (5) is not nullhomotopic. If we restrict the maps from $B D$ to $B V$ we obtain a diagram

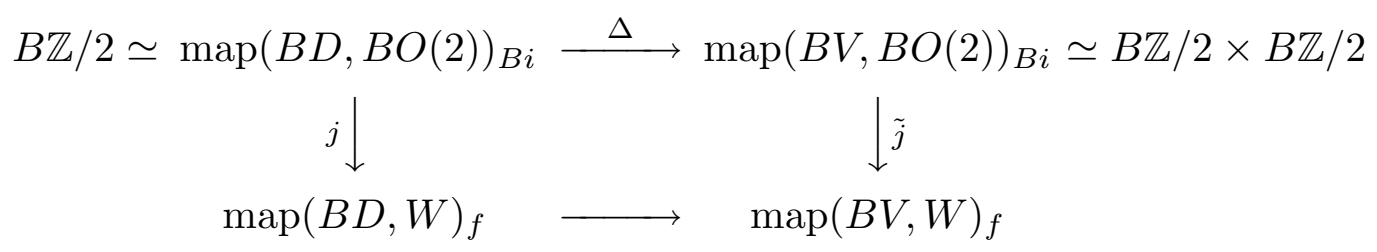

where we still denote by $B i$ and $f$ the restrictions of $B i: B D \rightarrow B O(2)$ and $f: B D \rightarrow W$ to $B V$ and $\Delta: B \mathbb{Z} / 2 \rightarrow B \mathbb{Z} / 2 \times B \mathbb{Z} / 2$ is the diagonal map.

Using Lannes $T$ functor we obtain

$$
H^{*}\left(\operatorname{map}(B V, W)_{f}\right) \cong \mathbb{F}_{2}\left[t_{1}, t_{2}\right] \otimes E\left(y_{2}\right)
$$


and $\tilde{j}$ induces in mod 2 cohomology the projection of $\mathbb{F}_{2}\left[t_{1}, t_{2}\right] \otimes E\left(y_{2}\right)$ onto $\mathbb{F}_{2}\left[t_{1}, t_{2}\right]$. Hence $\tilde{j}$ is non trivial at the level of the fundamental group. And then $j$ is non trivial either.

Standard arguments about fibrations show that

$$
\operatorname{map}\left(B \mathbb{Z} / 2, \operatorname{map}(B D, W)_{f}\right)_{j} \simeq \operatorname{map}(B D, W)_{f}
$$

A quick look at the exact sequences of homotopy groups of the fibrations in diagram (5) shows the existence of a section of the map $\operatorname{map}(B D, W)_{f} \rightarrow S^{2}$ that we can think of as being a map $S^{2} \rightarrow \operatorname{map}\left(B \mathbb{Z} / 2, \operatorname{map}(B D, W)_{f}\right)_{j}$. Taking the adjoint produces a diagram of fibrations

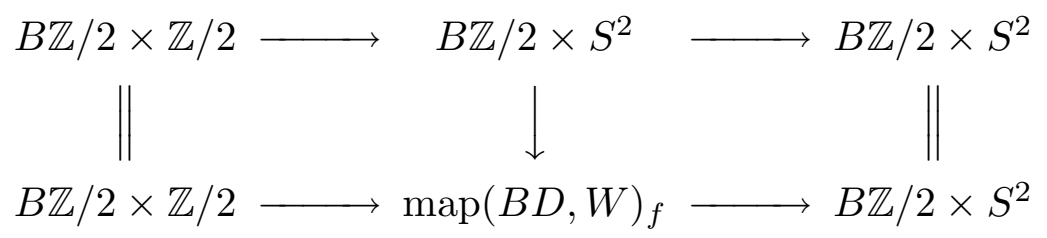

where the middle vertical arrow is a mod-2 equivalence. This proves the proposition.

Corollary 8. Let $B D \times S^{2} \rightarrow W$ be the adjoint of $S^{2} \rightarrow \operatorname{map}(B D, W)_{f}$. Then the restriction $B V \times S^{2} \rightarrow W$ is a map that in cohomology is

$$
\begin{aligned}
H^{*}(W) & \longrightarrow H^{*}(B V) \otimes H^{*}\left(S^{2}\right) \\
w_{1} & \longmapsto \sigma_{1} \\
w_{2} & \longmapsto \sigma_{2}+y_{2} \\
y_{3} & \longmapsto \sigma_{1} y_{2}
\end{aligned}
$$

Proof. The composition $B V \rightarrow B V \times S^{2} \rightarrow W$ is the obvious map and factors over $B O(2) \rightarrow W$ Hence, $w_{1} \mapsto \sigma_{1}$ and $w_{2} \mapsto \sigma_{2}+\lambda y_{2}$. The commutative diagram

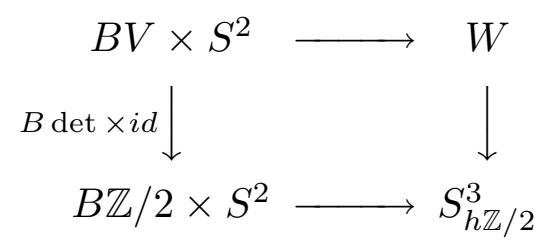

shows that $y_{3} \mapsto \sigma_{1} y_{2}$. The relation $S q^{1}\left(w_{2}\right)=w_{1} w_{2}+y_{3}$ finally shows that $w_{2} \mapsto$ $\sigma_{2}+y_{2}$.

\section{$\S 4$. THE SPACE $Y$.}

Let $I$ be the category associated to the symmetric group $\Sigma_{3}$ and the subgroup $\Sigma_{2}$ in the following way. $I$ has two objects 0 and 1 and the morphisms sets are

$$
\begin{aligned}
& \operatorname{Hom}(0,0)=\Sigma_{3} \\
& \operatorname{Hom}(0,1)=\emptyset \\
& \operatorname{Hom}(1,1)=\{1\} \\
& \operatorname{Hom}(1,0)=\Sigma_{3} / \Sigma_{2}
\end{aligned}
$$


with the obvious compositions given by the product in $\Sigma_{3}$ and the action of $\Sigma_{3}$ on $\Sigma_{3} / \Sigma_{2}$. This category was analised in the general case of a group $G$ and a subgroup $H$ in [2]. Let us consider the contravariant functor $\mathbf{M}: I \rightarrow \mathcal{U}$ given by

$$
\begin{aligned}
& M_{0}=H^{*}\left(B V \times S^{2}\right) \cong \mathbb{F}_{2}\left[t_{1}, t_{2}\right] \otimes E\left(y_{2}\right) \\
& M_{1}=H^{*}(W) \cong \mathbb{F}_{2}\left[w_{1}, w_{2}\right] \otimes E\left(y_{3}\right)
\end{aligned}
$$

with the natural action of $\Sigma_{3}=G L_{2} \mathbb{F}_{2}$ on $M_{0}$ and the homomorphism $\varphi: M_{1} \rightarrow M_{0}$ induced by the inclusion of $V$ as diagonal matrices in $D$. From the previous section we know that $\varphi$ is given by

$$
\begin{aligned}
& \varphi\left(w_{1}\right)=\sigma_{1} \\
& \varphi\left(w_{2}\right)=\sigma_{2}+y_{2} \\
& \varphi\left(y_{3}\right)=\sigma_{1} y_{2}
\end{aligned}
$$

\section{Proposition 9.}

$$
\stackrel{\lim }{I}^{i} \mathbf{M}=\left\{\begin{array}{l}
0, \quad i>0 \\
\mathbb{F}_{2}\left[\sigma_{1}^{2}+\sigma_{2}+y_{2}, \sigma_{1} \sigma_{2}\right] \otimes E\left(\sigma_{1} \sigma_{2} y_{2}\right), \quad i=0
\end{array}\right.
$$

Proof. The computation of the derived functors ${\underset{\lim }{\longleftarrow}}_{I}^{i}$ is done in [2]. For $i \geq 1$ there is a long exact sequence

$$
\cdots \rightarrow H^{i}\left(\Sigma_{3} ; M_{0}\right) \rightarrow H^{i}\left(\Sigma_{2} ; M_{0}\right) \rightarrow \overleftarrow{I}_{\lim ^{i+1}} \mathbf{M} \rightarrow H^{i+1}\left(\Sigma_{3} ; M_{0}\right) \rightarrow \cdots
$$

Since the restriction $H^{i}\left(\Sigma_{3} ; A\right) \rightarrow H^{i}\left(\Sigma_{2} ; A\right)$ is an isomorphism for any $i \geq 1$ and any $\mathbb{F}_{2}\left[\Sigma_{3}\right]$-module $A$ we obtain the vanishing of $\lim _{I}^{i+1} \mathbf{M}$ for any $i \geq 1$. To compute $\underset{\longleftarrow}{\lim _{I}^{0}} \mathbf{M}=$ $\left\{x \in M_{1} \mid \varphi(x) \in M_{0}^{\Sigma_{3}}\right\}$ notice that

$$
\begin{gathered}
\mathbb{F}_{2}\left[\sigma_{1}^{2}+\sigma_{2}+y_{2}, \sigma_{1} \sigma_{2}\right] \otimes E\left(\sigma_{1} \sigma_{2} y_{2}\right) \subset \underset{I}{\lim ^{0}} \mathbf{M} \\
\lim _{I}{ }^{0} \mathbf{M}=\left[\mathbb{F}_{2}\left[\sigma_{1}, \sigma_{2}+y_{2}\right] \otimes E\left(\sigma_{1} y_{2}\right)\right] \cap\left[\mathbb{F}_{2}\left[\sigma_{1}^{2}+\sigma_{2}, \sigma_{1} \sigma_{2}\right] \otimes E\left(y_{2}\right)\right] \\
\mathbb{F}_{2}\left[\sigma_{1}, \sigma_{2}\right] \otimes E\left(y_{2}\right)=\left[\mathbb{F}_{2}\left[\sigma_{1}, \sigma_{2}+y_{2}\right] \otimes E\left(\sigma_{1} y_{2}\right)\right]+\left[\mathbb{F}_{2}\left[\sigma_{1}^{2}+\sigma_{2}, \sigma_{1} \sigma_{2}\right] \otimes E\left(y_{2}\right)\right]
\end{gathered}
$$

Hence, the Poincaré series of $\lim _{I}{ }^{0} \mathbf{M}$ is given by the difference of the Ponjcare series of $\left[\mathbb{F}_{2}\left[\sigma_{1}, \sigma_{2}+y_{2}\right] \otimes E\left(\sigma_{1} y_{2}\right)\right] \oplus\left[\mathbb{F}_{2}\left[\sigma_{1}^{2}+\sigma_{2}, \sigma_{1} \sigma_{2}\right] \otimes E\left(y_{2}\right)\right]$ and of $\mathbb{F}_{2}\left[\sigma_{1}, \sigma_{2}\right] \otimes E\left(y_{2}\right)$. Comparing this with the Poincaré series of $\mathbb{F}_{2}\left[\sigma_{1}^{2}+\sigma_{2}+y_{2}, \sigma_{1} \sigma_{2}\right] \otimes E\left(\sigma_{1} \sigma_{2} y_{2}\right)$ calculates $\lim _{I}^{0} \mathbf{M}$. To obtain $\lim _{I}^{1} \mathbf{M}$ we use the exact sequence $([\mathbf{2}])$

$$
0 \rightarrow \lim _{I}{ }^{0} \mathbf{M} \rightarrow M_{1} \rightarrow Z \rightarrow \underset{I}{\lim _{I}^{1}} \mathbf{M} \rightarrow 0
$$


where $Z$ is isomorphic to the quotient $M_{0}^{\Sigma_{2}} / M_{0}^{\Sigma_{3}}$. Hence, the vanishing of $\lim _{I}^{1} \mathbf{M}$ can also be easily obtained by checking the agreement of some Poincaré series.

Next we want to construct a covariant functor $\mathbf{Y}: I \rightarrow \mathcal{S}_{*}$. We consider the exact sequence

$$
1 \rightarrow \mathbb{Z} / 2 \times \mathbb{Z} / 2 \cong V \rightarrow \Sigma_{4} \rightarrow \Sigma_{3} \rightarrow 1
$$

Then, the action of $\Sigma_{3}$ on $V$ is the standard one. We also get a right $\Sigma_{3}$-action on $B V \simeq E \Sigma_{4} \times_{\Sigma_{4}} \Sigma_{4} / V$ via the identification $\Sigma_{4} / V=\Sigma_{3}$ which induces the standard action on the fundamental group. The subgroup $V$ is contained in the 2-Sylow subgroup of $\Sigma_{4}$ which is isomorphic to $D$. Now we define

$$
\begin{aligned}
& Y_{0}=\left[E \Sigma_{4} \times_{\Sigma_{4}} \Sigma_{4} / V\right] \times S^{2} \simeq B V \times S^{2} \\
& Y_{1}=W .
\end{aligned}
$$

Then, the map

$$
\left[E \Sigma_{4} \times_{\Sigma_{4}} \Sigma_{4} / V\right] \times S^{2} \rightarrow\left[E \Sigma_{4} \times_{\Sigma_{4}} \Sigma_{4} / D\right] \simeq B D \times S^{2} \rightarrow W
$$

is $\mathbb{Z} / 2$-equivariant. The second is defined in the last section. Hence, this construction defines the desired functor $\mathbf{Y}: I \rightarrow \mathcal{S}_{*}$, which, up to homotopy, is the diagram mentioned the introduction:

$$
G L_{2}\left(\mathbb{F}_{2}\right) \quad \circlearrowright \quad B V \times S^{2} \quad \Longrightarrow W .
$$

Let $Y$ be the homotopy colimit of the functor $\mathbf{Y}$. Then the spectral sequence of BousfieldKan $([\boldsymbol{5}])$ and the vanishing of the higher derived functors of lim allow the computation of the cohomology of $Y$.

Corollary 10. $H^{*}(Y) \cong \mathbb{F}_{2}\left[z_{2}, z_{3}\right] \otimes E\left(y_{5}\right)$ and the map $\epsilon: W \rightarrow Y$ is such that $\epsilon^{*}\left(z_{2}\right)=$ $w_{1}^{2}+w_{2}, \epsilon^{*}\left(z_{3}\right)=w_{1} w_{2}+y_{3}, \epsilon^{*}\left(y_{5}\right)=w_{2} y_{3}$.

In particular, the action of the Steenrod algebra on $H^{*}(Y)$ is given by

$$
\begin{aligned}
& S q^{1} z_{2}=z_{3} \\
& S q^{2} z_{3}=z_{2} z_{3}+y_{5} \\
& S q^{2} y_{5}=z_{2} y_{5} \\
& S q^{1} y_{5}=S q^{4} y_{5}=0 .
\end{aligned}
$$

Because the classes $y_{5}$ and $z_{I}$ are mixed by the actiuon of the Steenrod algbera there is no fibration of the form $S^{5} \rightarrow Y \rightarrow B S O(3)$ or $B S O(3) \rightarrow Y \rightarrow S^{5}$.

\section{$\S 5$. THE SPACE $X$.}

Let now $X$ be the homotopy theoretic fibre of an essential map $Y \rightarrow B^{2} \mathbb{Z} / 2$. homotopy theoretic fibre 
Theorem 11. $H^{*}(X) \cong \mathbb{F}_{2}\left[x_{8}\right] \otimes E\left(S q^{1} x_{8}\right)$.

Proof. Notice that the map $k: X \rightarrow Y$ is trivial in cohomology. ¿From this it is easy to determine all the differentials in the Serre spectral sequence of the fibration

$$
B \mathbb{Z} / 2 \rightarrow X \rightarrow Y
$$

and one sees ea that the spectral sequence collapses at $E_{5}$ and

$$
E_{\infty}=E_{5} \cong \mathbb{F}_{2}\left[x_{8}\right] \otimes E\left(y_{9}\right) \text {. }
$$

It only remains to prove that $S q^{1} x_{8} \neq 0$. Consider the Serre spectral sequence of the fibration $X \rightarrow Y \rightarrow B^{2} \mathbb{Z} / 2$ and notice that

$$
\alpha=S q^{4} S q^{2} S q^{1} \iota+\iota^{2} S q^{2} S q^{1} \iota+\left(S q^{1} \iota\right)^{3} \in H^{*}\left(B^{2} \mathbb{Z} / 2\right)
$$

maps to zero in $H^{*}(Y)$. Hence, $x_{8} \in H^{*}(X)$ transgresses to $\alpha$. Then $S q^{1} x_{8}$ transgresses to $S q^{1} \alpha$ which is non-trivial in $H^{*}\left(B^{2} \mathbb{Z} / 2\right)$.

\section{§6. Non-Realizability of PE-algebras}

In this section we prove the main theorem stated in the introduction.

Let us assume $\mathbb{F}_{2}\left[x_{2 n}\right] \otimes E\left(S q^{1} x\right)$ is an unstable algebra over the Steenrod algebra. The classification of these algebras is done exactly as in the odd prime case $([\mathbf{3}])$. First one obtains the existence of the algebras

$$
\mathbf{A} \cong \mathbb{F}_{2}\left[x_{2}\right] \otimes E\left(y_{3}\right), \quad S q^{1}(x)=y, S q^{2}(y)=x y
$$

and

$$
\mathbf{B}_{i} \cong \mathbb{F}_{2}\left[x_{2^{i+1}}\right] \otimes E\left(y_{2^{i+1}+1}\right), \quad S q^{1}(x)=y, S q^{2^{i+1}}(y)=0
$$

as algebras over the Steenrod algebra and then one proves that there are no more.

Proposition 12. Let $A$ be a $P E$-algebra which is an unstable algebra over the Steenrod algebra and such that $S q^{1}(x)=y$. Then $A$ is isomorphic, as an algebra over the Steenrod algebra, to one of the algebras $\mathbf{A}, \mathbf{B}_{i}$ as defined above.

Proof. Since the ideal generated by $y$ is closed under the Steenrod algebra action the degree of $x$ should be a power of two.

In the case when $i>0$ the Adem relation

$$
S q^{2^{i}} S q^{2^{i}+1}=\sum_{j=0}^{2^{i-1}} \mu_{j} S q^{2^{i+1}+1-j} S q^{j}
$$

gives $S q^{2^{i+1}} y=0$ when applied to $x$. Hence $A$ should be isomorphic to $\mathbf{B}_{i}$. If $i=0$ then either $S q^{2} y=0$ or $S q^{2} y=x y$ and $A$ is isomorphic to $\mathbf{B}_{0}$ and $\mathbf{A}$, respectively. 
Let $X$ be the space constructed in the previous sections. We have $H^{*}(X) \cong \mathbf{B}_{2}$. Let $F$ be the space considered in section 1 . Then

$$
\begin{aligned}
H^{*}(F) & \cong \mathbb{F}_{2}\left[x_{2}\right] \otimes E\left(S q^{1} x_{2}\right)=\mathbf{B}_{0} \\
H^{*}\left(S^{3}\langle 3\rangle\right) & \cong \mathbb{F}_{2}\left[x_{4}\right] \otimes E\left(S q^{1} x_{4}\right)=\mathbf{B}_{1}
\end{aligned}
$$

Hence the "if" part of the main theorem is proved.

Remark. The spaces $F, S^{3}\langle 3\rangle$ and $X$ are not the unique ones realizing $\mathbf{B}_{0}, \mathbf{B}_{1}$ and $\mathbf{B}_{2}$ respectively, not even up to 2-completion. In [4] we construct infinite families of spaces which realize these cohomology algebras. Actually, the goal of [4] is a complete classification of all 2-complete homotopy types realizing $\mathbf{B}_{0}, \mathbf{B}_{1}$ and $\mathbf{B}_{2}$.

It only remains to prove

Theorem 13. (Cooke conjecture for $p=2[\mathbf{6}],[\mathbf{3}]$.) Assume that there is a space $X$ with $H^{*}(X) \cong \mathbf{B}_{i}$. Then $i=0,1$ or 2 .

Proof. The proof is similar to the proof of the Cooke conjecture in $[\mathbf{3}]$ except for some details in small dimensions. We can as well assume that $X$ is 2-complete because $H^{1}(X)=$ 0 . From $[8]$ we know that there is a map

$$
\phi: B \mathbb{Z} / 2 \rightarrow X
$$

inducing the non trivial $f_{0}: \mathbf{B}_{i} \rightarrow H^{*}(B \mathbb{Z} / 2)$ and such that, according to the computation of the value of the $T$ functor on the algebra $\mathbf{B}_{i}$ done in $[\mathbf{3}]$, the evaluation

$$
\operatorname{map}(B \mathbb{Z} / 2, X)_{\phi} \rightarrow X
$$

is a homotopy equivalence. Now $B \mathbb{Z} / 2$ acts on this mapping space and if $Y$ denotes the homotopy quotient, we obtain a sequence of fibrations:

$$
B \mathbb{Z} / 2 \stackrel{j}{\rightarrow} \operatorname{map}(B \mathbb{Z} / 2, X)_{\phi} \rightarrow Y \rightarrow B^{2} \mathbb{Z} / 2
$$

where $j: B \mathbb{Z} / 2 \rightarrow \operatorname{map}(B \mathbb{Z} / 2, X)_{\phi}$ composed with the evaluation becomes $\phi$.

Our results arise from the analysis of the Serre spectral sequence for computing the cohomology of $Y$. This gives us the necessary restrictions on the dimensions of the generators of the cohomology of $X$.

Recall that $H^{*}\left(B^{2} \mathbb{Z} / 2\right)$ is a polynomial algebra on the generators

$$
\iota, S q^{1} \iota, S q^{2} S q^{1} \iota, \ldots, S q^{\Delta_{j}} \iota, \ldots
$$

where we use the notation $S q^{\Delta_{j}}=S q^{2^{j}} S q^{2^{j-1}} \ldots S q^{1}$. Observe that the Adem relations and instability imply that the following relations hold in $H^{*}\left(B^{2} \mathbb{Z} / 2\right)$ :

$$
\begin{aligned}
& S q^{1} S q^{\Delta_{j}} \iota=\left(S q^{\Delta_{j-1}} \iota\right)^{2} \quad j>0 \\
& S q^{2} S q^{\Delta_{j}} \iota=0 \quad j>1
\end{aligned}
$$


The first observation is that since the evaluation is an equivalence $j$ looks like $\phi$ in cohomology: $j^{*}(x)=u^{2^{i+1}}$ and therefore in the Serre spectral sequence for $\operatorname{map}(B \mathbb{Z} / 2, X)_{\phi} \rightarrow$ $Y \rightarrow B^{2} \mathbb{Z} / 2$ the class $x$ transgresses to $S q^{\Delta_{i}}$ plus decomposables:

$$
\tau(x)=S q^{\Delta_{i}} \iota+d .
$$

Assume now $i>2$. In the Serre spectral sequence we have the transgressions:

$$
\begin{aligned}
& \tau(x)=S q^{\Delta_{i}} \iota+d \\
& \tau(y)=S q^{1}\left(S q^{\Delta_{i}} \iota\right)+S q^{1} d=\left(S q^{\Delta_{i-1}} \iota\right)^{2}+S q^{1} d
\end{aligned}
$$

Now, if we apply Steenrod operations to either $\tau(x)$ or $\tau(y)$ we obtain new elements in the bottom line of the spectral sequence that have to be killed by a differential. Let us try with $S q^{2}$ applied to $\tau(y)$. Since $S q^{2}\left(S q^{\Delta_{i-1}} \iota\right)^{2}=\left(S q^{\Delta_{i-2}} \iota\right)^{4}$ we obtain that $S q^{2} \tau(y)=$ $\left(S q^{\Delta_{i-2} \iota}\right)^{4}+S q^{2} S q^{1} d$ has to be zero modulo elements killed by a differential. Let us denote $\alpha:=\tau(x)$ and $\beta:=\tau(y)$. Then our equation is

$$
0=\left(S q^{\Delta_{i-2}} \iota\right)^{4}+S q^{2} S q^{1} d \quad \bmod (\alpha, \beta)
$$

Notice that $d$ can be written as

$$
d=a S q^{\Delta_{i-1}} \iota+b S q^{\Delta_{i-2}} \iota+c
$$

where $a, b$ and $c$ are polynomials in $\iota, S q^{1} \iota, \ldots, S q^{\Delta_{i-3}} \iota$. Hence, a straightforward argument shows that equation (1) cannot be solved if $i>3$. If $i=3$ then the equation (1) admits several solutions because in that case $S q^{2} S q^{1}$ applied to either $S q^{\Delta_{0}} \iota \cdot S q^{\Delta_{1}} \iota, S q^{\Delta_{2}} \iota$ or $\iota\left(S q^{\Delta_{1}} \iota\right)^{3}$ gives a term $\left(S q^{\Delta_{1}} \iota\right)^{4}$. Nevertheless, $d$ should also satisfy other equations obtained by applying $S q^{2^{k}}$ to $\alpha$ and $\beta$. In particular we obtain:

$$
S q^{2}(d)=S q^{4}(d)=0 \quad \bmod (\alpha, \beta)
$$

and a long and boring but straightforward computation shows that no one of the solutions of (1) can satisfy these new equations.

Remark. In [4] we prove that $\mathbf{A}$ is also realizable as the mod two cohomology of some space.

\section{$\S 7$. The Arf invariant}

In [1], [3], [4] and the present paper we have considered spaces $X$ whose mod $p$ cohomology has the form $\mathbb{F}_{p}\left[x_{2 n}\right] \otimes E(\beta x)$ where $\beta$ denotes the Bockstein homomorphism. One sees immediately a relation between these spaces and the Arf invariant elements. For instance, (see also [1]) assume that $X$ is 2-complete and

$$
H^{*}(X) \cong \mathbf{B}_{i-1}=\mathbb{F}_{2}\left[x_{2^{i}}\right] \otimes E\left(S q^{1} x_{2^{i}}\right) .
$$


Then the cell structure of $X$ looks like

$$
X=S^{2^{i}} \cup_{2} e^{2^{i}+1} \cup_{\theta} e^{2^{i+1}} \cup \cdots
$$

Here we use the standard notation in which $\theta \in \pi_{2^{i+1}-1}\left(S^{2^{i}+1}\right)$ is the map obtained from the attaching map of $e^{2^{i+1}}$ by collapsing the $2^{i}$-skeleton. Let $X^{\prime}$ be the Spanier-Whitehead dual of the $2^{i+1}$-skeleton of $X$. The cohomology of $X^{\prime}$ has generators $a, b$ and $c$ linked by Steenrod operations in the way $S q^{2^{i}} a=S q^{1} b=c$. Then, for $i>3$ the Adams decomposition of $S q^{2^{i}}$ through secondary operations implies that $a$ and $b$ have to be linked by the secondary operation corresponding to the Arf invariant elements in the stable stem. Hence, a realization of $\mathbf{B}_{i}, i>2$, would immediately provide an Arf invariant element. But theorem 13 in the present paper shows that $\mathbf{B}_{i}, i>2$ is not realizable as the cohomology of any space. Therefore, although Arf invariant elements have been constructed up to the 62-stem, only $\eta^{2}$ and $\nu^{2}$ could be used to build a space with cohomology $\mathbf{B}_{i}$. The siapplying is similar to what happens with the Hopf invariant one elements. Any space whose cohomology is a polynomial algebra on one generator should have the second cell attached to the first one through a map of Hopf invariant one but, although $\eta, \nu$ and $\sigma$ have Hopf invariant one, only $\eta$ and $\nu$ can be the starting block of such a space.

If $X$ realizes $\mathbf{B}_{1}$ or $\mathbf{B}_{2}$ then the above argument cannot be used to show that the third cell is attached by the Arf invariant element, because $S q^{4}$ and $S q^{8}$ are not decomposable through secondary operations. Actually, we do not know what is the attaching map of the third cell of the space $X$ constructed in section 5 . In the case of $S^{3}\langle 3\rangle$ the attaching map for the third cell is indeed $\eta^{2}$.

Proposition 14. $S^{3}\langle 3\rangle_{2}=S^{4} \cup_{2} e^{5} \cup_{\eta^{2}} e^{8} \cdots$.

Proof. It suffices to prove that the 8-cell is attached non-trivially to the 5-cell. Let us first recall the few first stable homotopy groups of $B S^{1}$ at the prime two (cf. [9]):

$$
\begin{array}{cccccccc}
i= & 1 & 2 & 3 & 4 & 5 & 6 & 7 \\
\pi_{*}^{S}= & 0 & \mathbb{Z} & 0 & \mathbb{Z} & \mathbb{Z} / 2 & \mathbb{Z} & \mathbb{Z} / 2 \\
\text { generator } & & \beta_{1} & & 2 \beta_{2} & \beta_{1} \nu & 2 \beta_{3} & \beta_{2} \nu
\end{array}
$$

We then use the homology Serre spectral sequence of the fibration $B S^{1} \rightarrow S^{3}\langle 3\rangle \rightarrow S^{3}$ to compute the stable homotopy of $S^{3}\langle 3\rangle$ :

$$
E_{p, q}^{2}=H_{p}\left(S^{3} ; \pi_{q}^{S}\left(B S^{1}\right)\right) \quad \Rightarrow \quad \pi_{p+q}^{S}\left(S^{3}\langle 3\rangle\right) .
$$

¿From this one deduces that

$$
\pi_{7}^{S}\left(B S^{1}\right) \rightarrow \pi_{7}^{S}\left(S^{3}\langle 3\rangle\right)
$$

is an isomorphism. Since the generator of $\pi_{7}^{S}\left(B S^{1} / S^{2}\right)$ comes from the fourth-dimensional cell, we obtain that $\pi_{7}^{S} S^{4}=\mathbb{Z} / 8 \nu$ projects to $\pi_{7}^{S}\left(S^{3}\langle 3\rangle\right)$. Consider the diagram

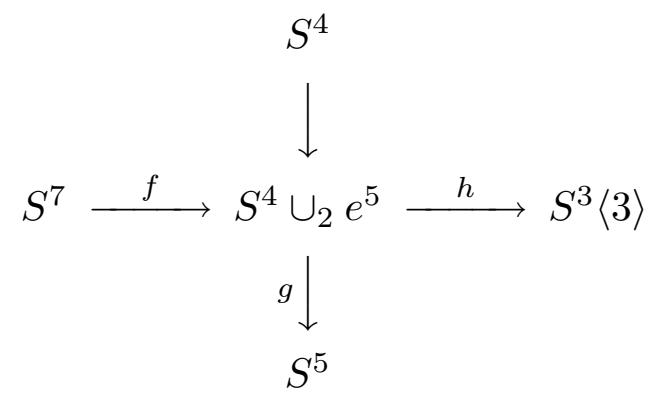


We want to prove that $g f$ is essential. If not, then $f$ lifts stably to $S^{4}$ but, since $h f$ is trivial, $f$ is divisible by two in $\pi_{7}^{S} S^{4}$ and so it is stably trivial in $\pi_{7}^{S}\left(S^{4} \cup_{2} e^{5}\right)$. Since $f$ is the attaching map of the seven-dimensional cell of $S^{3}\langle 3\rangle$, this contradicts the existence of a non-trivial $S q^{4}$ in $S^{3}\langle 3\rangle$.

We finish with some open questions which deserve further study.

The above proof relies on the map $S^{3}\langle 3\rangle \rightarrow S^{3}$ and so it does not work if $S^{3}\langle 3\rangle$ is replaced by a space with the same cohomology but a different mod 2 homotopy type. All such spaces are classified in [4] and it would be interesting to know if the attaching map of the third cell of such a space should always be $\eta^{2}$.

In the present paper we have constructed a space

$$
X=S^{8} \cup_{2} e^{9} \cup_{\theta} e^{16} \cup \cdots
$$

whose cohomology is $\mathbf{B}_{2}$. Is $\theta=\nu^{2}$ ? In a similar way as in the case of $S^{3}\langle 3\rangle$, the $\bmod 2$ homotopy types of all spaces with the same cohomology than $X$ are classified in [4]. If $X^{\prime}$ is now one of these spaces, is also the third cell of $X^{\prime}$ attached through the Arf invariant map?

\section{REFERENCES}

[1] J. Aguadé, Cohomology algebras with two generators, Math. Z. 177 (1981), 289-296.

[2] J. Aguadé, Constructing Modular Classifying Spaces, Israel J. Math. 66 (1989), 23-40.

[3] J. Aguadé, C. Broto and D. Notbohm, Homotopy Classification of Some Spaces With Interesting Cohomology and a Conjecture of Cooke, Part I, To appear in Topology.

[4] J. Aguadé, C. Broto and D. Notbohm, Homotopy Classification of Some Spaces With Interesting Cohomology and a Conjecture of Cooke, Part II, in preparation.

[5] A. Bousfield and D. Kan, Homotopy limits, completions and localizations, Lecture Notes in Math. 304, Springer Verlag, 1972.

[6] G. Cooke, Constructing spaces with interesting $\mathbb{Z} / p$-cohomology via $\epsilon$-actions on loop spaces, Amer. J. Math. 101 (1979), 515-542.

[7] W.G. Dwyer and A. Zabrodsky, Maps between classifying spaces, Proceedings of the 1986 Barcelona Conference on Algebraic Topology, Lecture Notes in Math. 1298, Springer Verlag, 1988.

[8] J. Lannes, Sur les espaces fonctionnels dont la source est le classifiant d'un p-groupe abélien élémentaire, Publ. Math. IHES 75 (1992), 135-244.

[9] A. Liulevicius, A theorem in homological algebra and stable homotopy of projective spaces, Trans. Amer. Math. Soc. 109 (1963), 540-552.

J. Milnor, Constructions of universal bundles, Ann. Math. 63 (1956), 272-284.

[11] Notbohm, Maps between classifying spaces and applications, To appear in Jour. Pure Appl. Algebra.

[12] J. Stasheff, Homotopy associativity of H-spaces, Trans. Am. Math. soc. 108 (1963), 275-292.

Departament de Matemàtiques, Universitat Autònoma de Barcelona, 08193 Bellaterra, SPAIN.

Mathematisches Institut Der Georg August Universität Göttingen, Bunsenstrasse 3, 37073 Göttingen, Germany. 